\title{
Optimization of the Belle II Vertex Detector
}

\section{Zbynek DRASAL*i}

Charles University Prague, Czech Republic

E-mail: drasaleipnp.troja.mff.cuni.cz

\section{Kolja PROTHMANN}

Max-Planck-Institut für Physik München, Germany

E-mail: kolja@mpp.mpg.de

At the high luminosities envisaged for the new Super Flavour Factory - SuperKEKB, the detection system closest to the beam pipe - a new Belle II silicon vertex detector will have to face an extremely harsh beam-related background and, consequently, high hit event rates, but still provide precise measurements of primary and secondary decay vertices. During the design phase of the vertex detector, consisting of two layers of pixel sensors and four layers of double-sided microstrip sensors, one has to carefully take into account all available technological options and optimise the detector design to fully reach the expected physics performance. For that reason we have initially utilized a modular design of ILC software framework and used it in full MC simulations for multi-parameter studies. In this paper we present the description of the Belle II vertex detector, our approach to the multi-parameter optimisation studies and finally, the obtained results: the detailed study of the expected material distributions of the vertex detector; optimisation study of the pixel detector layout with the final determination of the most optimal configuration; the influence of the slanted microstrip detectors in the forward region on the detector in-plane resolution and hit occupancy; and the expected impact parameter resolutions using or not using the two innermost pixel layers (to demonstrates the improvement of impact parameter resolutions by implementation of pixel sensors at Belle II). For consistency, the software chain has been validated against the official Belle software framework and cosmic muons data taken at Belle.

The XIth International Conference on Heavy Quarks and Leptons,

June 11-15, 2012

Prague, Czech Republic

\footnotetext{
* Speaker.

${ }^{\dagger}$ This work has been supported by the Czech Science Foundation Grant No. 203/10/0777 and the Ministry of Education, Youth and Sports of the Czech Republic No. LA10033 and MSM0021620859.

$¥$ This work has been supported by the DFG cluster of excellence "Origin and Structure of the Universe” of Germany.
} 


\section{Introduction}

SuperKEKB, an upgrade of the successful asymmetric $\mathrm{e}^{+} \mathrm{e}^{-}$collider and actual world luminosity record holder $\left(2.11 \times 10^{34} \mathrm{~cm}^{-2} \mathrm{~s}^{-1}\right)$ KEKB (Tsukuba, Japan), will start commissioning by fall 2014 [1]. The main goal of the new Super Flavour Factory is to dramatically increase instantaneous statistics by a factor of 40 and thus deliver an integrated luminosity of $50 \mathrm{ab}^{-1}$ by end of 2022. For that reason, the nano-beam option has been chosen. The beam pipe radius in the interaction region will be then only about $10 \mathrm{~mm}$, which has a positive effect on the physics related to vertex reconstruction, but it presents a big challenge for the vertex detector itself due to harsh background environment. The background level generally increases roughly as the inverse square of the radius. In Belle, the innermost layers of the vertex detector consisted of microstrip sensors. But due to the expected large hit occupancy and, consequently, increased amount of false 2D hits, so-called ghosts, the innermost layers of the new precision vertex detector can no longer be composed of microstrip sensors and pixel sensors have to be used instead. The pixel detectors have much larger number of read-out channels, and thus lower occupancy, and will provide hits with true 2D information (unlike the strips combining two pieces of 1D information into a common hit, which is ambiguous). Furthermore, as low momentum particles ( $\sim$ several hundreds of MeV) dominate at B-factories, multiple Coulomb scattering plays an important role in vertex reconstruction. Therefore, the material budget of the vertex detector, particularly of the innermost part, has to be seriously constrained. The pixel detector concept, based on the Depfet [月] technology, allows for such low material budget.

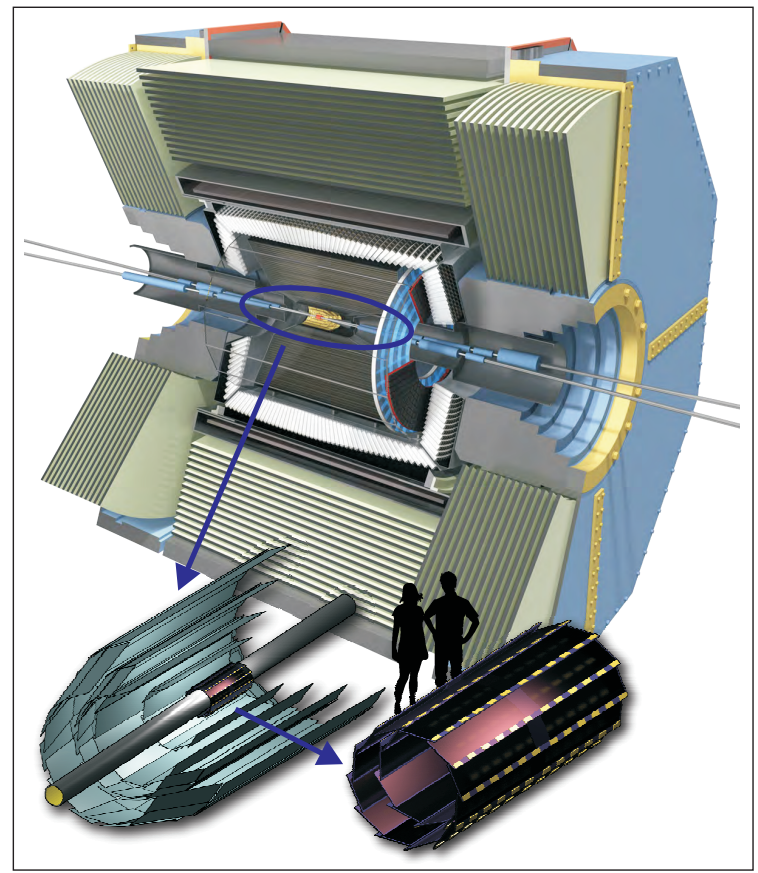

Figure 1: A scheme of the future Belle II detection system with a detail of the silicon vertex detector. The SVD together with PXD and a beam pipe is shown on the left, the standalone PXD on the right.

In order to deal with the challenging working conditions, particularly the increased background level and higher event rates, and, simultaneously, provide the required high precision measurements 
of B meson decay vertices, a new design of the Belle II silicon VerteX Detector (VXD) is planned, see Fig. 1. The vertex detector will consist of 2 layers of thinned $(75 \mu \mathrm{m})$ Depfet-type pixel detectors (PXD), with radii of 14 and $22 \mathrm{~mm}$, and 4 layers of double-sided microstrip detectors (SVD), with radii of 38, 80, 115 and $140 \mathrm{~mm}$. The three outermost layers of SVD are extended to the forward region (slanted design) to fully cover, together with a central drift chamber (CDC), Belle II angular acceptance from $17^{\circ}$ to $150^{\circ}$ in polar angle.

\section{Multi-Parameter Optimization Study \& Software Framework}

During the initial phase of each vertex detector project one has to carefully take into account the different aspects of detector technical design, such as layout, materials, cooling, etc., and choose the optimal configuration to fully achieve the planned physics performance. An ideal tool for such multi-parameter optimisation studies are simulations, allowing to try out all available design options. For that reason we have initially utilized a modular design of ILC software framework [3] and adapted its functionality to the Belle II needs. In the software framework we have implemented the full chain of the following tools: generator (Evtgen and KoralW for generation of Belle physics events and expected QED background respectively), Geant4 simulation - Mokka (with full material implementation of VXD geometry), digitization (pixel and microstrip silicon detectors simulation [四). Furthermore, we have utilized the ILC software pattern recognition and adapted the tracking software (Marlin modules). For detailed scheme of the simulation chain see Fig. 2. In the simulations, the influence of following parameters (configurations) has been studied: material budget, layer radius, layer thickness, pixel geometry arrangements, pixel size, geometry setup in the forward region.

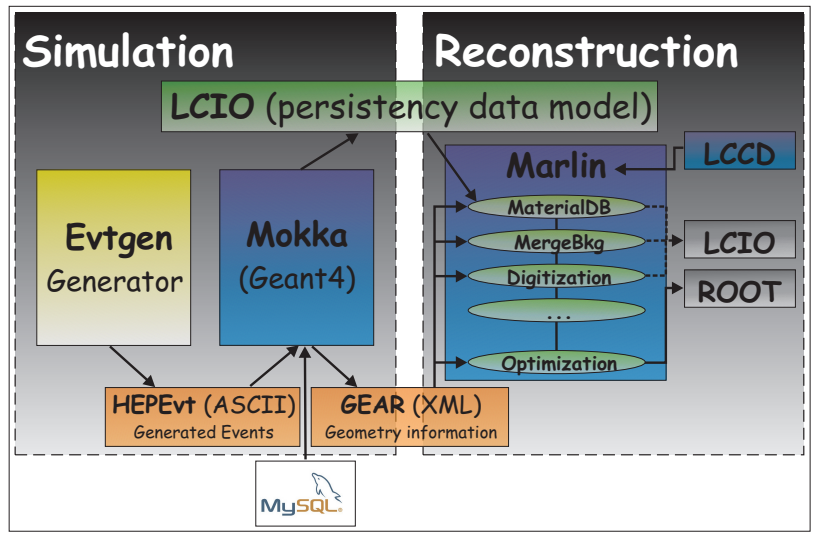

Figure 2: The scheme of software simulation and reconstruction chain (based on ILC software framework).

\subsection{The Expected Material Budget of the VXD}

The expected material distributions of the whole vertex detector have been studied using Geant4 simulations for two different options (Fig. 3). One with a barrel-like geometry configuration in the forward region, the other one with a conical-like geometry, i.e. with SVD with slanted sensors in the forward part. The latter represents the current Belle II baseline configuration. 


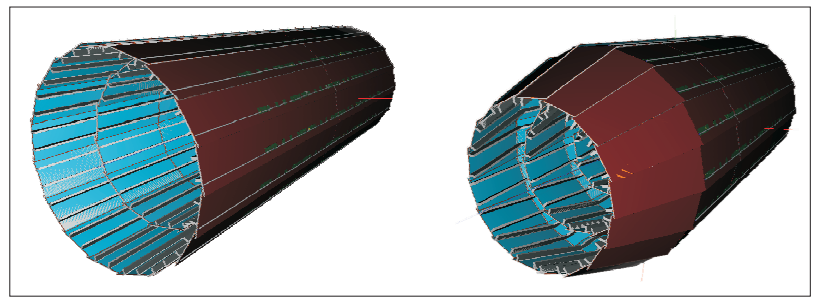

Figure 3: Vertex detector geometry in two options, with the barrel-like geometry in the forward region (barrel-like SVD), depicted on the left, the other one with the conical-like geometry in the forward region (conical-like SVD), depicted on the right.

The following key components have been implemented: a beryllium beam pipe with a $10 \mu \mathrm{m}$ thick golden foil (meant as a protection against soft synchrotron radiation) and a cooling gap filled with paraffin as a cooling medium, active PXD silicon sensors together with their passive parts (silicon rims, support and electronics chips, so-called switchers), and, finally, active SVD silicon sensors together with their passive parts (sandwich carbon fiber ribs, thermal and eletrical insulation (Rohacell layer), kaptons, read-out chips and a steel cooling pipe filled with $\mathrm{CO}_{2}$ as a cooling medium). The parameters of the baseline geometry, used in the simulations, are summarized in Tab. 1, the results are shown in Fig. 月. Comparing the two options one can clearly see the substantial increase in material budget for the SVD barrel-like geometry in the region from $17^{\circ}$ to $45^{\circ}$, which grows up by $50 \%$.

\begin{tabular}{lcccc}
\hline & R [mm] & \#Ladders & \#Sensors & $\vartheta[\mathbf{d e g}]$ \\
\hline PXD layer 1 & 14 & 8 & 2 & 0.0 \\
PXD layer 2 & 22 & 12 & 2 & 0.0 \\
SVD layer 1 & 38 & 8 & 2 & 0.0 \\
SVD layer 2 & 80 & 10 & 3 & 11.9 \\
SVD layer 3 & 115 & 14 & 4 & 17.2 \\
SVD layer 4 & 140 & 17 & 5 & 21.1 \\
\hline
\end{tabular}

Table 1: The parameters of Belle II baseline geometry with slanted detectors in the forward region.

\subsection{Optimization Study of the Depfet Pixel Layout}

The Depfet pixel sensors can be manufactured in several layout options. The final physics performance, for different options, can be efficiently studied with single particles ( $0.5 \mathrm{GeV}$ muons), generated at the unsmeared $e^{+} e^{-}$interaction point with selected values of polar angle $\theta$ and uniformly distributed in azimuth $\phi$. For evaluation, we have used the quantity of the in-plane (intrinsic) resolution in both sensor directions: in $R$ - $\Phi$ plane (perpendicular to the beam) and $Z$ direction (parallel to the beam). The first option, Fig. 5, have been a study of constant (CPS) versus variable (VPS) pixel size in $Z$ direction. The study has been performed for two configurations: with 800 pixels in $Z$ ( $p_{1} \sim 123 \mu \mathrm{m}, p_{2} \sim 147 \mu \mathrm{m}$ for CPS and $p_{1} \sim 117-178 \mu \mathrm{m}, p_{2} \sim 145-174 \mu \mathrm{m}$ for VPS, where $p$ stands for the pitch of the first and second layer respectively) and with 1600 pixels 

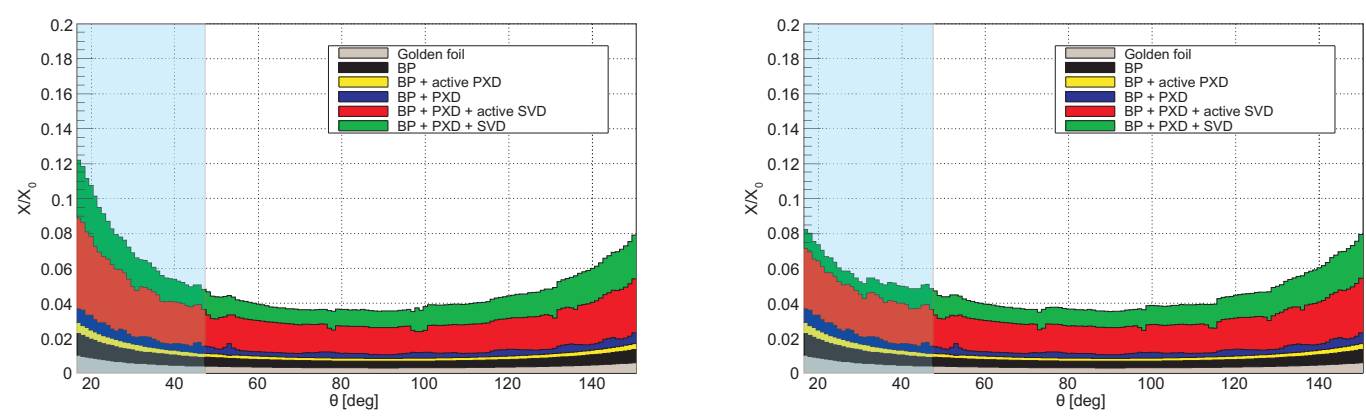

Figure 4: Expected material distributions obtained from Geant4 simulation: SVD barrel-like geometry on the left, SVD conical-like geometry on the right. The individual bands correspond to: the beam pipe golden foil, beryllium beam pipe and cooling gap filled with paraffin, active PXD sensors, passive PXD material (silicon rims, support and electronics chips), active SVD sensors, passive SVD material (sandwich carbon fiber ribs, Rohacell layer, kaptons, read-out chips and the steel cooling pipe filled with $\mathrm{CO}_{2}$.

in $Z$, the baseline, $\left(p_{1} \sim 61 \mu \mathrm{m}, p_{2} \sim 73 \mu \mathrm{m}\right.$ for CPS and $p_{1} \sim 38-177 \mu \mathrm{m}, p_{2} \sim 56-174 \mu \mathrm{m}$ for VPS). The key idea of the variable pixel size consists in keeping the cluster size (and thus resolution) constant along the $Z$-axis, independently on the polar angle of emitted particles. This can be practically done by decreasing the pixel size gradually from the edges of each ladder to the point closest to the interaction point. So, we have first geometrically evaluated (using a line from the interaction region) the optimal pixel size starting at the edges of the ladder, further we have kept on decreasing the pitch until we reached the minimal size, limited by the given number of pixels in $Z$. The minimal pixel size has been used then for the remaining pixels to the point (on the ladder) closest to the interaction point. For details, see schematic drawing in Fig.6.
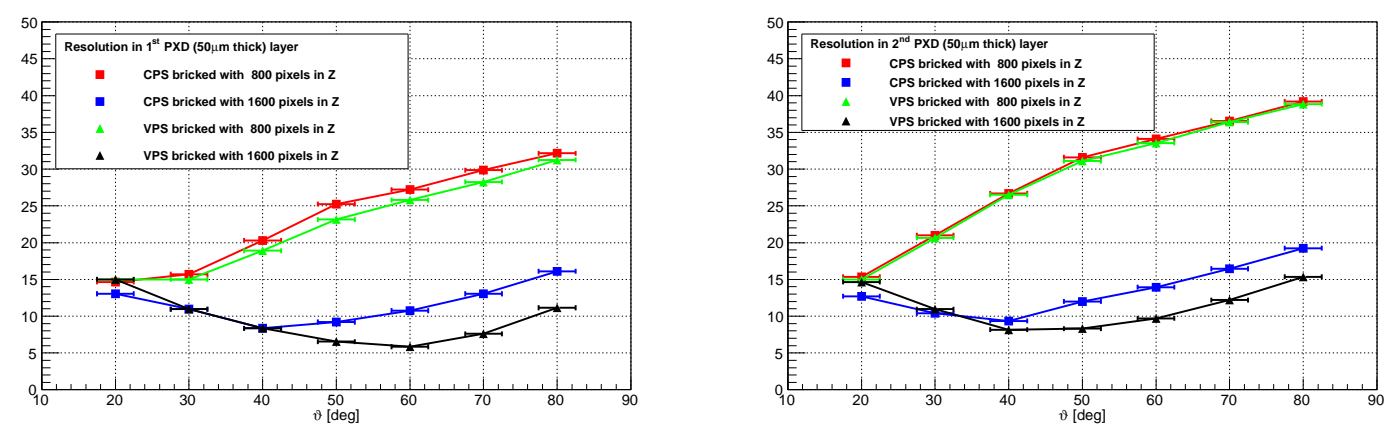

Figure 5: PXD in-plane resolution $[\mu \mathrm{m}]$ in $Z$ direction for different design options: CPS with bricked structure, $50 \mu \mathrm{m}$ pitch in $R$ - $\Phi$ and 800 pixels in $Z$ (filled red squares); CPS with bricked structure, $50 \mu \mathrm{m}$ pitch in $R$ - $\Phi$ and 1600 pixels in $Z$ (filled blue squares); VPS with bricked structure, $50 \mu \mathrm{m}$ pitch in $R$ - $\Phi$ and 800 pixels in $Z$ (filled green triangles); VPS with bricked structure, $50 \mu \mathrm{m}$ pitch in $R$ - $\Phi$ and 1600 pixels in $Z$ (filled black triangles). The resolution for the first layer is depicted on the left side, for the second layer on the right side. PXD thickness has been set to $50 \mu \mathrm{m}$.

The studies have been performed for $50 \mu \mathrm{m}$ thick PXD sensors, the originally considered design, and the in-plane resolution has been defined as a root mean square of the $95 \%$ area of in- 


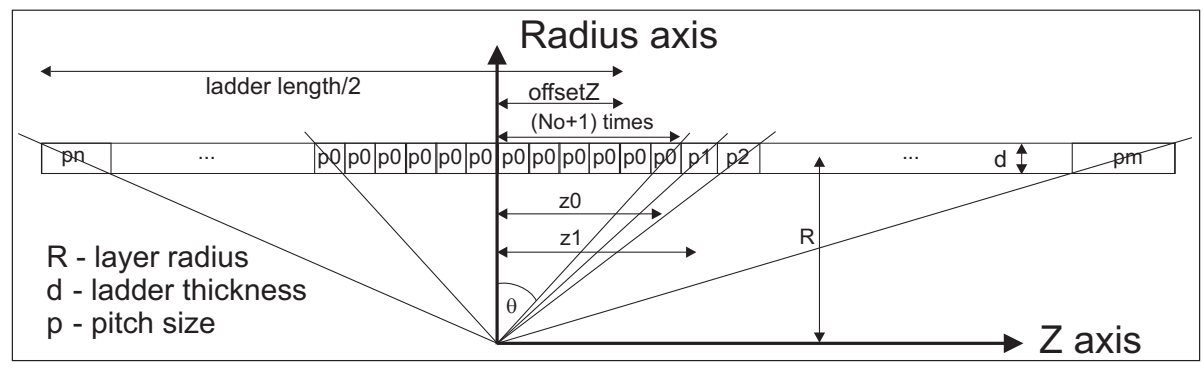

Figure 6: The schematic drawing of VPS option. The $p_{0}$ parameter corresponds to the pitch of smallest pixels, repeated $(N+1)$ times, then the pitch increases continuously until the ladder edge is reached. Due to the asymmetry of ladder position with respect to the interaction point, the pitch on the left is of different size than on the right.

plane signal residuals distribution. Such a definition has excluded non-gaussian tails and provided good stability independently on the polar and azimuthal angle of reconstructed particles.

The second line of optimisation studies, Fig.7, has been the study of bricked versus nonbricked structure in $R-\Phi$ plane, where the bricked stands for a layout with pixels shifted by a half of pitch every even row. The key idea of bricked structure consists in increasing signal/noise ratio for edge pixels in clusters of size higher than one in $Z$, and thus increasing resolution for nonperpendicular tracks. The pixel pitch has been set to two sizes: $p \geq 50 \mu \mathrm{m}$ for unbricked structure and $p \geq 70 \mu \mathrm{m}$ for bricked structure (lower not technologically achievable).

Due to the technological limits on pixel pitch (bricked technology available for $p \geq 70 \mu \mathrm{m}$ only) and expected gain in resolution limited to the forward region only, the unbricked layout has been preferred. The variable pixel size brings significant improvement in resolution in the area where most of the produced particles fly (due to B-factory boost), but requires internal implementation of extra drift fields in Depfet (too large pixels at the edges of sensors would lead to inefficiencies in charge collection), which complicates the pixel design. So, the Depfet baseline has been finally fixed to: $75 \mu \mathrm{m}$ thick PXD with CPS along $Z$-axis and unbricked structure in $R$ $\Phi$ plane and will consist of: $2 \times 768$ rows in $Z(256 \times 55 \mu \mathrm{m}+512 \times 60 \mu \mathrm{m}$ for the first layer, $256 \times 70 \mu \mathrm{m}+512 \times 85 \mu \mathrm{m}$ for the second layer), 250 columns $\times 50 \mu \mathrm{m}$ pitch in $R$ - $\Phi$.

\subsection{Optimization Study of the SVD Forward Region}

Due to the asymmetry of energy of colliding particles at the SuperKEKB factory, the SVD design with slanted sensors in the forward region brings a significant reduction in terms of the number of needed sensors to fully cover the required Belle II acceptance region. On the other hand, the mechanical design and consequently, mechanical stability, alignment, etc. are more challenging than for the same geometry, but with extended (barrel-like) sensors in the forward region. In order to see the difference in terms of material budget (see section 2.1), detector in-plane resolution (defined as a root mean square of the $90 \%$ area of signal residuals distribution) and final hit occupancy (correlated with a cluster size and important for efficiency of charged particles reconstruction), we have studied the cluster size (Fig. 8) and in-plane resolution (Fig. 9) in $Z$ direction. There is no difference between the geometries in $R$ - $\Phi$ plane. 

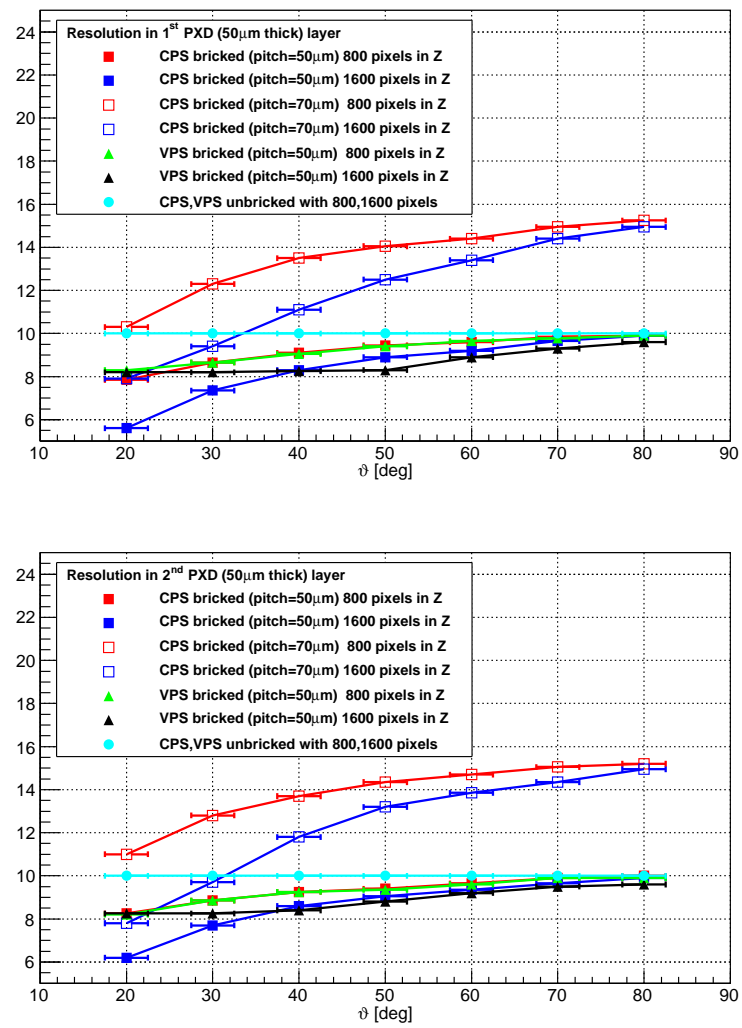

Figure 7: PXD in-plane resolution $[\mu \mathrm{m}]$ in $R$ - $\Phi$ direction for different design options: CPS with bricked structure, $50 \mu \mathrm{m}$ pitch in $R$ - $\Phi$ and 800 pixels in $Z$ (filled red squares); CPS with bricked structure, $50 \mu \mathrm{m}$ pitch in $R$ - $\Phi$ and 1600 pixels in $Z$ (filled blue squares); CPS with bricked structure, $70 \mu \mathrm{m}$ pitch in $R$ - $\Phi$ and 800 pixels in $Z$ (open red squares); CPS with bricked structure, $70 \mu \mathrm{m}$ pitch in $R$ - $\Phi$ and 1600 pixels in $Z$ (open blue squares); VPS with bricked structure, $50 \mu \mathrm{m}$ pitch in $R$ - $\Phi$ and 800 pixels in $Z$ (filled green triangles); VPS with bricked structure, $50 \mu \mathrm{m}$ pitch in $R$ - $\Phi$ and 1600 pixels in $Z$ (filled black triangles); and CPS or VPS with unbricked structure, $50 \mu \mathrm{m}$ pitch in $R$ - $\Phi$ and 800 or 1600 pixels in $Z$ (filled cyan circles). The resolution for the first layer is depicted on the top, for the second layer at the bottom. PXD thickness has been set to $50 \mu \mathrm{m}$.
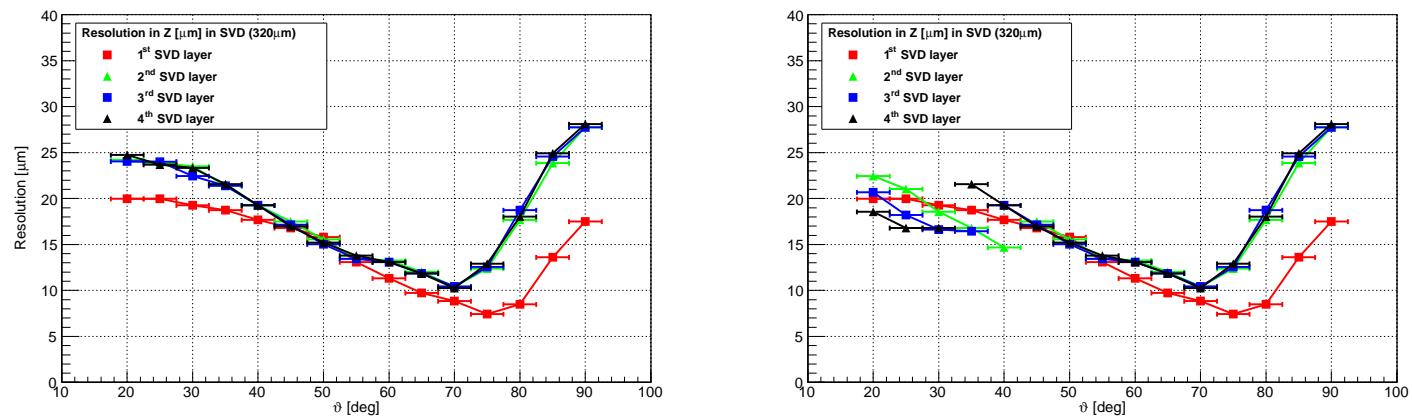

Figure 8: SVD in-plane resolution $[\mu \mathrm{m}]$ in $Z$ for different vertex detector geometry options: SVD barrellike (left) and SVD conical-like (right). The first layer is depicted with red, second with green, third with blue and fourth with black colour. SVD sensors are $320 \mu \mathrm{m}$ thick. 

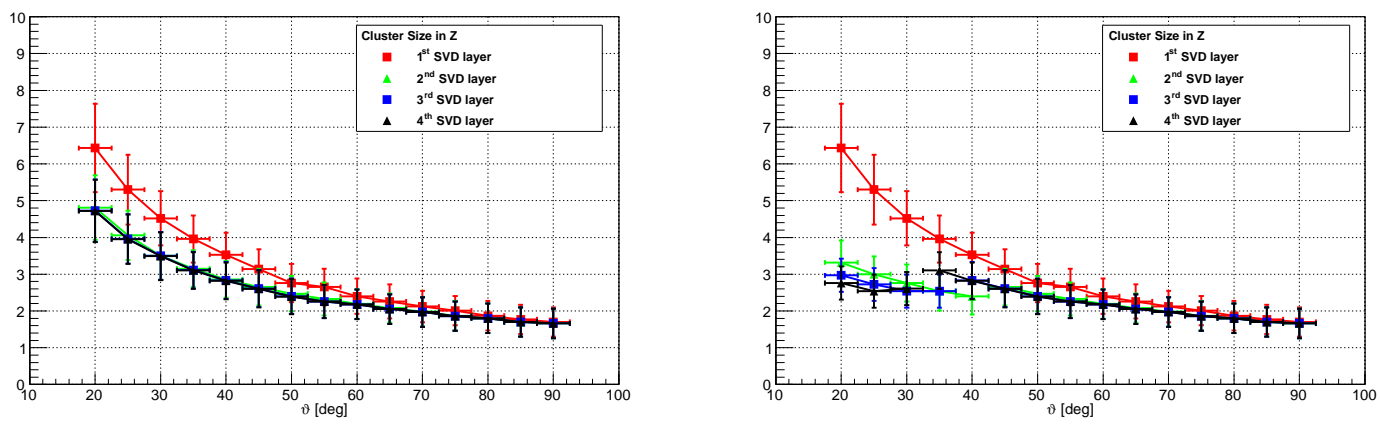

Figure 9: SVD cluster size in $Z$ direction for different vertex detector geometry options: SVD barrel-like (left) and SVD conical-like (right). The first layer is depicted with red, second with green, third with blue and fourth with black colour. SVD sensors are $320 \mu \mathrm{m}$ thick.

\subsection{Impact Parameter Studies}

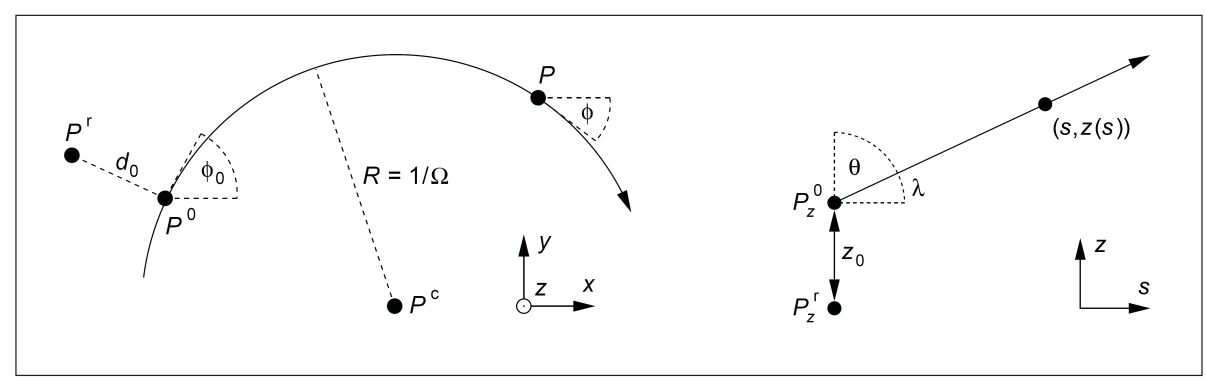

Figure 10: Definition of impact parameters in $R-\Phi$ plane $-d_{0}$ and in $s-Z$ plane $-z_{0}$, where $s$ denotes the length of the trajectory arc in $R-\Phi$ plane. All parameters are expressed with respect to the reference point $P^{\mathrm{r}}$, the point of closest approach is denoted as $P^{0}$.

The resolution of impact parameters (IP) $-d_{0}$ and $z_{0}$, defined (Fig.10) as the projections of distance from the point of closest approach $P^{0}$ to the reference point $P^{\mathrm{r}}$ (identical to the origin for single particle studies and to the vertex for decay studies), are a good measure of the overall performance of the tracking system and are used to find the optimal tracker (vertex detector) configuration. The main reason is that the parameters naturally test the interplay between the geometrical setup of individual sensitive detectors and physics effects, particularly multiple Coloumb scattering and ionization losses. The mathematical formula describing the IP resolution $\sigma$ can be then written as a sum of two terms: intrinsic detector resolution and the term related to multiple Coloumb scattering, which depends on particle momenta:

$$
\sigma=\sqrt{a^{2}+b^{2} / \tilde{p}^{2}} \quad \text { often written as } \quad \sigma=a \oplus b / \tilde{p}
$$

, where

$$
\begin{aligned}
& \tilde{p}=p \beta \sin \vartheta^{3 / 2} \quad \text { stands for } \quad d_{0} \\
& \tilde{p}=p \beta \sin \vartheta^{5 / 2} \quad \text { stands for } z_{0}
\end{aligned}
$$


More details about how $d_{0}$ and $z_{0}$ are defined in the ILC software framework can be found in [5].

In order to validate the whole software chain in the ILC software framework, we have first studied the IP resolution $\sigma\left(z_{0}\right)$ for positive muons reconstructed from the $\mathrm{J} / \Psi$ decay (coming from the decay of neutral B-mesons) in both BASF [6] and ILC framework. (For that purpose we have implemented the Belle detector geometry.) Further, we have compared achieved results with the distributions from Belle cosmic muons measurements [7]. The IP resolution curves are depicted in Fig. 11 and clearly demostrate a very good agreement between the Belle standard simulation tool BASF and the new software used here.

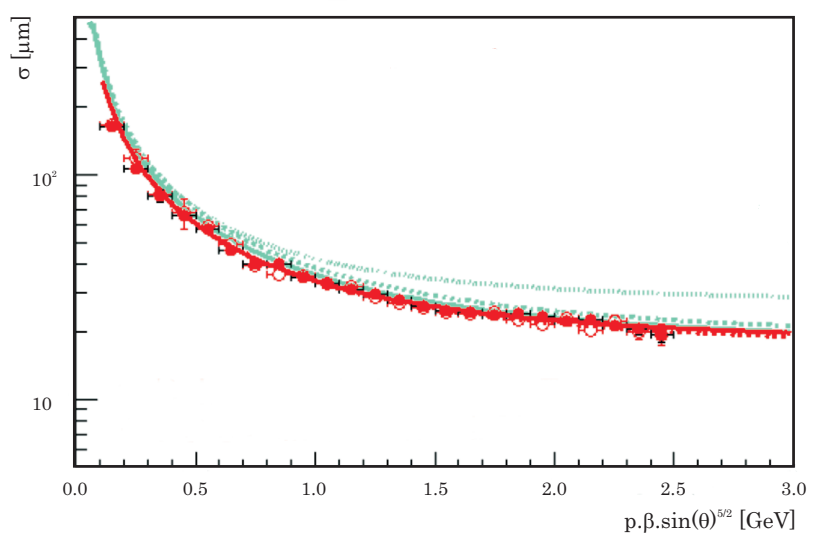

Figure 11: IP resolution $-\sigma\left(z_{0}\right)$ for positive muons reconstructed from $\mathrm{J} / \Psi$ decay in BASF (red open circles, red dashed curve) and ILC software (red filled circles, red solid curve). In addition, the resolutions from Belle data/MC cosmic muons are depicted: data (dotted cyan curve - upper curve), Monte Carlo in BASF (dashed cyan curve - lower curve).

Finally, we have studied the expected performance of the Belle II vertex detector: PXD+SVD for typical Belle II particle energies, see the resolution region below $1 \mathrm{GeV}$ in Fig. 12 and compared obtained results with the performance of SVD only, Fig. 12. The IP resolution has been defined as the sigma of double gaussian core (for fitter stability), where the double gaussian has been fitted in the $90 \%$ area, the non-gaussian (exponential) tails have been excluded. The obtained results clearly demonstrate the substantial improvement in IP resolution due to the installation of PXD at Belle II:

Parameters for PXD+SVD:

$$
\begin{aligned}
& \sigma\left(d_{0}\right)=(9.7 \pm 0.5) \oplus(11.1 \pm 0.7) \mu \mathrm{m} / \tilde{p} \\
& \sigma\left(z_{0}\right)=(12.1 \pm 0.5) \oplus(17 \pm 1) \mu \mathrm{m} / \tilde{p}
\end{aligned}
$$

Parameters for SVD only:

$$
\begin{aligned}
& \sigma\left(d_{0}\right)=(13.8 \pm 0.9) \oplus(37 \pm 2) \mu \mathrm{m} / \tilde{p} \\
& \sigma\left(z_{0}\right)=(15 \pm 1) \oplus(44 \pm 2) \mu \mathrm{m} / \tilde{p}
\end{aligned}
$$



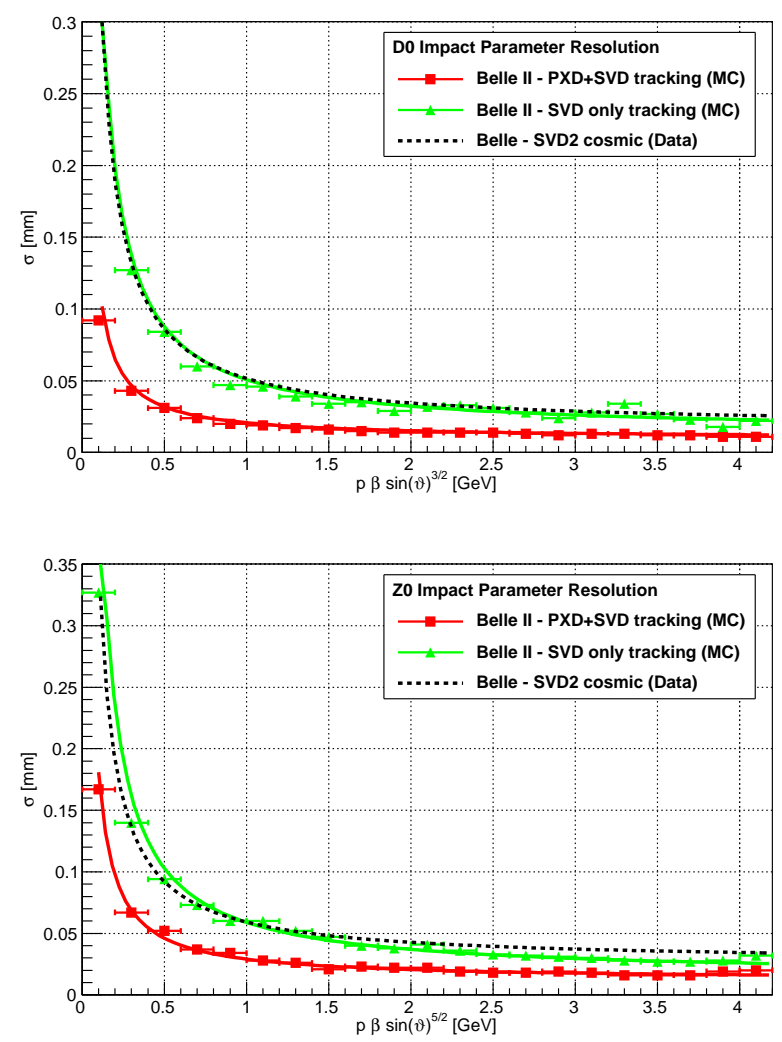

Figure 12: The impact parameter resolution in $R$ - $\Phi$ plane - $\sigma\left(d_{0}\right)$ (on the left) and in $Z$ direction - $\sigma\left(z_{0}\right)$ (on the right) for Belle II detector geometry. Two options have been simulated: results from the combined PXD and SVD tracking (red squares) and from SVD only tracking (green triangles). For comparison, in dashed curve, the resolution of Belle vertex detector (SVD2), obtained from the cosmic data, has been depicted.

For comparison, the resolution of Belle vertex detector (SVD - version 2) [7], [8] has been depicted. It clearly shows how the PXD detector improves the IP resolution also compared to the Belle experiment.

\section{Summary}

For the optimisation studies of the Belle II experiment we have developed a full simulation, digitization, pattern recognition and tracking chain in the ILC software framework. We have validated its functionality comparing impact parameter resolutions with those obtained in the Belle software framework (BASF). Concerning the optimisation results, we have studied the expected material budget of the future vertex detector (Belle II PXD and SVD) and significantly helped to understand the resolution of the new pixel and micrstrip detectors. Moreover, we have contributed to the final determination of the Depfet pixel layout. And finally, we have studied the improvement of impact parameter resolutions due to the installation of PXD vertex detector at the Belle II experiment and compared the results with those of a vertex detector consisting of SVD only. 


\section{References}

[1] Belle II collaboration, Belle II Technical Design Report, arXiv: 1011.0352, KEK 2010

[2] DEPleted Field Effect Transistor, [http://www. depfet.org]

[3] ILC Software, official web page, [http://ilcsoft.desy.de/portal]

[4] Z. Drasal, K. Prothmann, B. Schwenker, Silicon Simulation Code for Belle II and ILC, PoS (Vertex 2011), 027

[5] T. Krämmer, Track Parameters in LCIO, [www-flc.desy.de/lcnotes/notes/LC-DET-2006-004.pdf]

[6] BASF, official Belle Analysis Software, [http://belle.kek.jp]

[7] H.Ishino et al., Alignment Method for the SVD2 and Its Performance, internal Belle note BN715

[8] H.Aihara et al., Belle SVD2 vertex detector, Nuclear Instruments and Methods in Physics Research A (2006), 568, issue 1, p. 269-273 